\title{
Gedanken über den Sexualzyklus
}

Prof. Dr. med. Karl Junkmann.

Herr Praesident, meine Damen und Herren!

Lassen Sie mich zu Beginn meines Vortrages wiederum meiner Freude Ausdruck geben ueber die grosse Ehre, die es fuer mich bedeutet, hier vor Thnen zu sprechen. Ich habe als Thema meines Vortrages Gedanken ueber den Sexualcyclus gewaehlt und moechte Ihnen eigene Beobachtungen und einige bekannte Tatsachen aus der Literatur vor Augen fuehren und die Gedanken schildern, die ich daran geknuepft habe. Es mag sein, dass diese Gedanken in mancher Beziehung irrig sind, sie moegen jedoch dazu beitragen, das verwickelte Problem des Zusammenspiels der mit dem Zyclus beschaeftigten Hormone zu klaeren. Darf ich an den Beginn meiner Ausfuehrungen die heutige Lehrmeinung ueber das Zusammenspiel der Hormone beim Zyklus stellen.

\begin{tabular}{|c|c|c|}
\hline \multicolumn{2}{|c|}{ Hypophysenvorderlappen HVL } & Ovar \\
\hline $\begin{array}{l}\text { Follikelstimulierendes } \\
\text { Hormon } \\
\qquad \text { FSH }\end{array}$ & $\begin{array}{l}\text { reift die Follikel } \\
\text { an ,ohne sie zur } \\
\text { Sekretion zu be- } \\
\text { fähigen }\end{array}$ & \multirow[b]{2}{*}{$\begin{array}{l}\text { Östrogen, Ö, wirkt } \\
\text { auf die sekundä- } \\
\text { ren Geschlechts- } \\
\text { merkmale fördernd } \\
\text { und hemmt die } \\
\text { Sekretion von hy- } \\
\text { pophysärem Gona- } \\
\text { dotropin. Fördert } \\
\text { die Sekretion von } \\
\text { LTH durch den HVL. }\end{array}$} \\
\hline $\begin{array}{l}\text { Interstitialzellstimu- } \\
\text { lierendes Hormon } \\
\text { ICSH }\end{array}$ & $\begin{array}{l}\text { beendet Follikel- } \\
\text { reife, führt zu } \\
\text { Thecaluteinisi- } \\
\text { rung und zur Östro- } \\
\text { genproduktion des } \\
\text { Follikels }\end{array}$ & \\
\hline $\begin{array}{l}\text { Luteotrophes Hormon }= \\
\text { Prolakt in } \\
\text { LTH }\end{array}$ & $\begin{array}{l}\text { beendet die Reife } \\
\text { des Corpus luteum } \\
\text { und befähightes } \\
\text { zur Sekretion von } \\
\text { Progesteron }\end{array}$ & $\begin{array}{l}\text { Gestagen (Proge- } \\
\text { steron), G ,be-- } \\
\text { wirkt progestio-- } \\
\text { nale Umwandlung } \\
\text { der durch Ôstro- } \\
\text { gen proliferier-- } \\
\text { ten Uterusschleim- } \\
\text { haut. }\end{array}$ \\
\hline
\end{tabular}

Man nimmt an, dass das Follikelstimulierende Hormon FSH im Ovarium die Follikel anreift, dass eine nachfolgende Sekretion des Interstitialzellen stimulierenden Hormons ICSH die Reifung der Follikel beendet, ihre Luteinisierung einleitet und den Follikel befaehigt, Follikelhormon zu sezernieren. Dieses Follikelhormon wuerde auf dem Umweg ueber ein Sexualzentrum, oder direkt auf die Hypophyse einwirkend, zur Sekretion von Luteotrophen Hormon oder Prolaktin auch LTH genannt fuehren, das die Reifung des Corpus Luteum beendet und die Sekretion von Lutechormen ermoeglichen wuerde. Darf ich Sie nun auf einige Versuche aufmerksam machen, die wir 
in der Zwischenzeit angestellt haben.

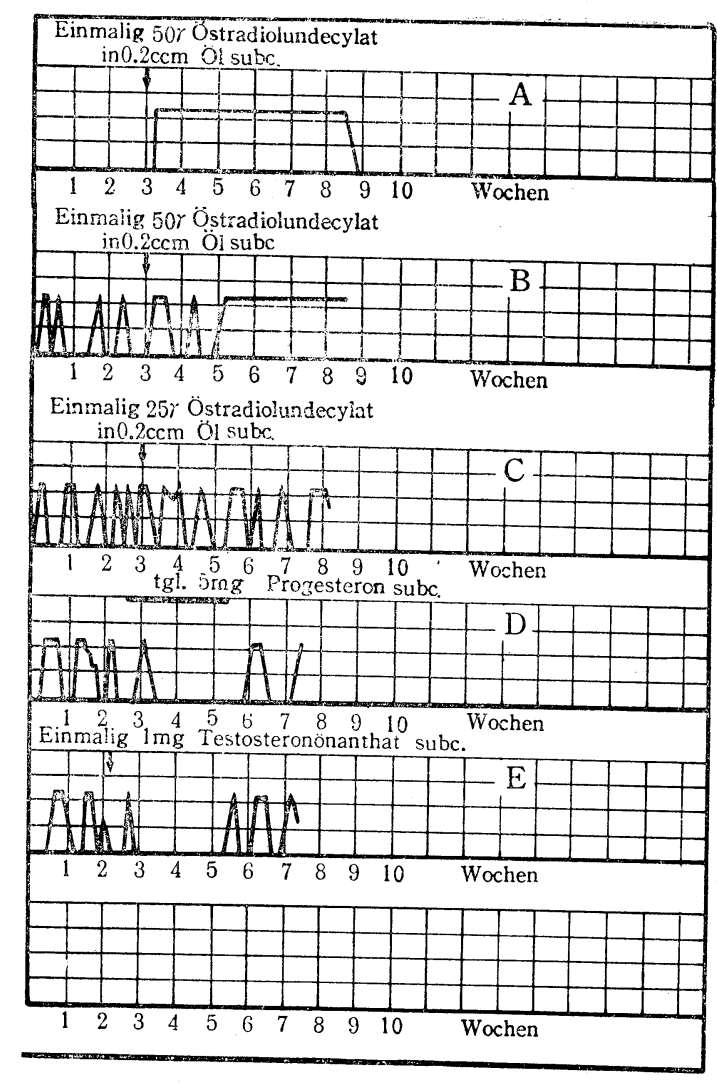

Abbildung 2 zeigt Ihnen das Verhalten des Vaginaloestrus einer kastrierten Ratte nach der Injektion von Oestradiolundecylat, einem sehr lang protrahiert wirksamen Oestrogen. Nach einmaliger Gabe von 25 tritt ein Oestrus von wenigstens 40 Tagen Dauer ein. Versucht man dasselbe bei einer nicht kastrierten Ratte, so stellt sich heraus, dass der Oestruszyclus dieser Ratte keineswegs unterbrochen wird. Kastriert man nach Ablauf einiger weiterer Zyklen unter der Oestradioleinwirkung, so tritt der merkwuerdige Fall ein, das auf die Kastration ein Daueroestrus erfolgt. Es ist also das Follikelhormon in dieser Ratte wirksam gewesen, nur hat das Ovar rythmisch die Wirkung des Daueroestrus unterbrochen. Es waere nun vielleicht daran zu denken, dass es das Corpus luteum ist, das diese rhytmische Unterbrecheung der Oestradiolundecylatdauerwirkung bewerkstelligt. Leider gelingt es nicht durch physiologische Dosen Progesteron den Daueroestrus unter 25 Oestradiolundecylat zu unterbrechen. Ich darf nebenbei erwaehnen, dass diese Unterbrechung nur eine scheinbare ist und dass es sich nicht um eine antioestrogene Wirkung des Progesterons handelt sondern dass das Progesteron die cornifizierte Schleimhaut der Scheide muzifiziert. Ganz anders ist es mit Testosteron. Mit Testosteron gelingt es, den Daueroestrus durch Oestradiolundezylat schon mit 
relativ kleinen Dosen zu unterbrechen. Es lag daher nahe, die merkwuerdige Erscheinung, dass es nicht gelingt, den normalen Zyklus der Ratte durch relativ hohe Gaben Oestradiolundezylat in einen Daueroestrus zu verwandeln, auf einen Androgenzyclus zurueckzufuehren. Dass das Ovar Androgene produziert, ist eine nicht unbekannte Tatsache. Ich erinnere nur an die virilisierenden Ovarialtumoren. Dauf ich ihr Augenmerk nunmehr auf eine zweite Erscheinung richten. Wenn wir eine infantile Ratte hypophysektomieren und ihr nach einer genuegend langen Wartezeit von wenigstens 8-14 Tagen Choriongonadotropin oder Stutenserumgonadotropin injizieren, so beobachten wir im Ovar nicht die gewohnten Erscheinungen der Follikelreifung und der Corpus
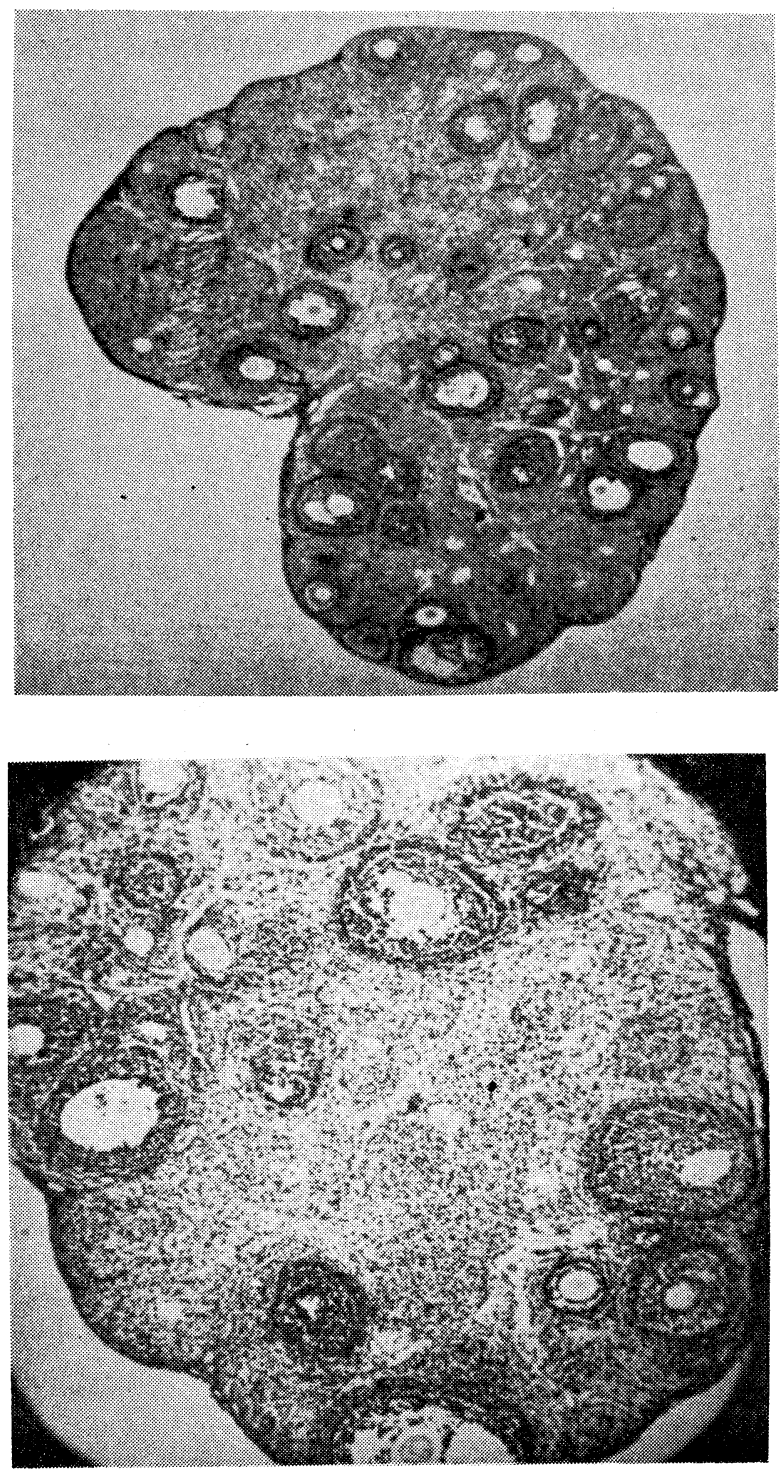

Vol. 31 No. 5 
luteumbildung. Das einzige, was ein solches Over nach laengeror taeglichen Injektion von Choriongonadotropin oder Stuenserumgonadotropin zeigt, ist eine maessige Gewichtzunahme, bedingt durch eine maechtige Hypertrophie der interstitiellen Zellen. Sie sehen dies in der naechsten Abbildung 3 und in staerkerer Vergroesserung ebenfalls in der Abbildung 4. Die Follikel sind unreif und hoechstens eine unbedeutende Huellenbildung in einzelnen Follikeln zu sehen, jedenfalls keine volle Follikelreifung. Wir wenden uns nunmehr einer dritten Tatsache zu. Injiziert man weiblichen kastrierten Ratten Oestradiolvalerianat und beobachtet das Uterusgewicht und das Gewicht der Praeputialdruesen, so stellt sich wie bekannt heraus, dass das Uterusgewicht bedeutend zunimmt, dass andererseits das Gewicht der Praeputialdruesen unveraendert niedrig bleibt, wie es die folgende Abbildung zeigt (4a). Die Praeputialdruesen werden also

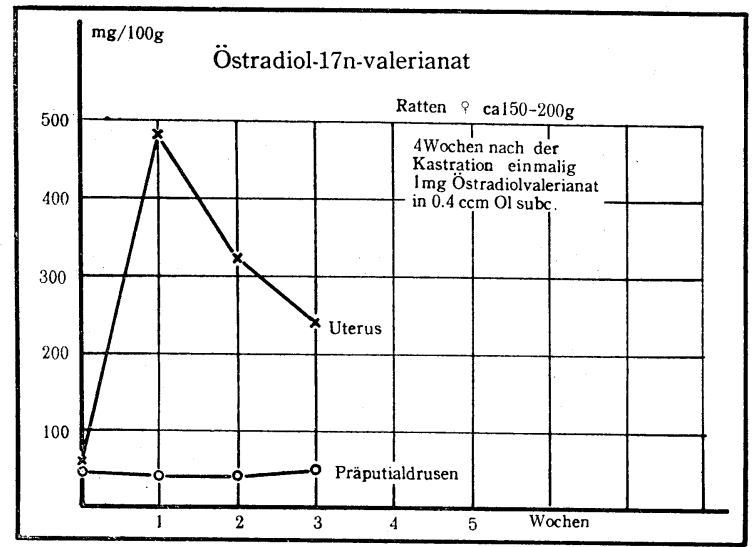

durch Oestrogene nicht stimuliert. Injiziert man dagegen kastrierten weiblichen Ratten Testosteronoenenthat in einer einmaligen grossen Dosis, so wird das Uterusgewicht wie bekannt maessig stimuliert, die Praeputialdruesen werden jedoch deutlich aktiviert. Die Praeputialdruesen sind demnach bei der weiblichen Ratte ein androgenstimuliertes Organ. Ich darf hinzufuegen, dass die Praeputialdruesen durch Progesteron nicht beeinflusst werden, dass sie dagegen im besten Fall in ihrer Atrophie nach der Kastration gehemmt werden, wenn man Progesteron in hohen Dosen anwendet. Weiter ergibt sich, dass man auch einer infantilen Ratte Testosteronpropionat injizieren und damit eine Steigerung des Praeputialdruesengewichts erreichen kann, wie dies aus der naechsten Abbildung 5 hervorgeht. Schliesslich zeigt auch die naechste Abbildu'g 6 , dass auch die Injektion von Choriongonadotropin an der infantilen Ratte nebon der bekannten Gewichtssteigerung des Uterus und der Ovarien zu einer deutlichen Steigerung des Praeputialdruesengewichtes, also zu einer androgenen Stimulierung der Ovarien fuehrt. Das gleiche konnte, wie die naechste Abbildung 7 zeigt auch mit Stutenserumgonadotropin nachgewiesen werden. Hier sehen wir die starke Stimulierung des Ovargewichts, die relativ starke Steigerung des Uterusgewichts und eine deutliche 

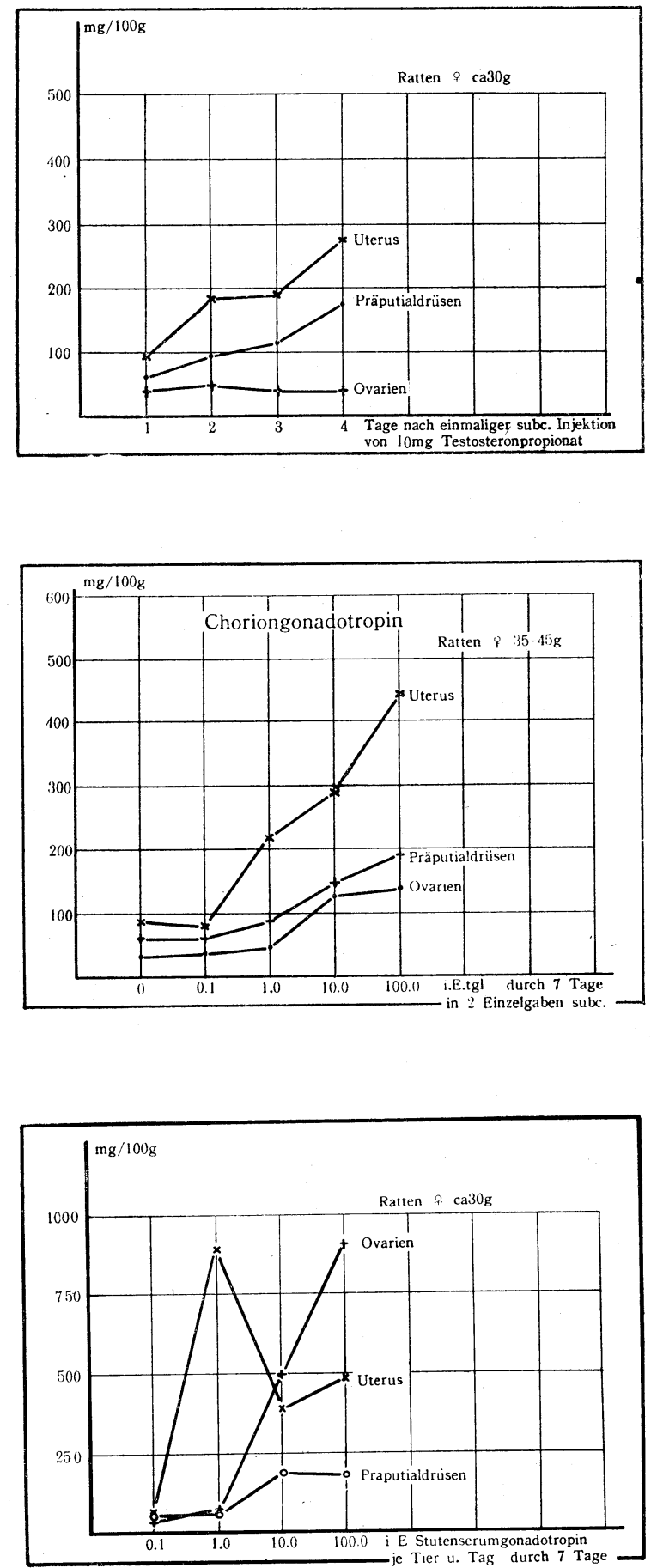

Vol. 31 No. 5 
Erhoehung des Gewichtes der Praeputialdruesen. Alle diese Tatsachen zusammen machten mir den angenommenen androgenen Zyclus im Ovar immer mehr wahrscheinlich. Ich habe mich nun natuerlich bemueht am Praeputialdruesengewicht im Verlauf des normalen Rattenzyklus auch hier einen Zyklus nachweisen zu koennen. Das zeigt Ihnen die folgende Abbildung 8. Leider war es am Praeputialdruesengewicht nicht

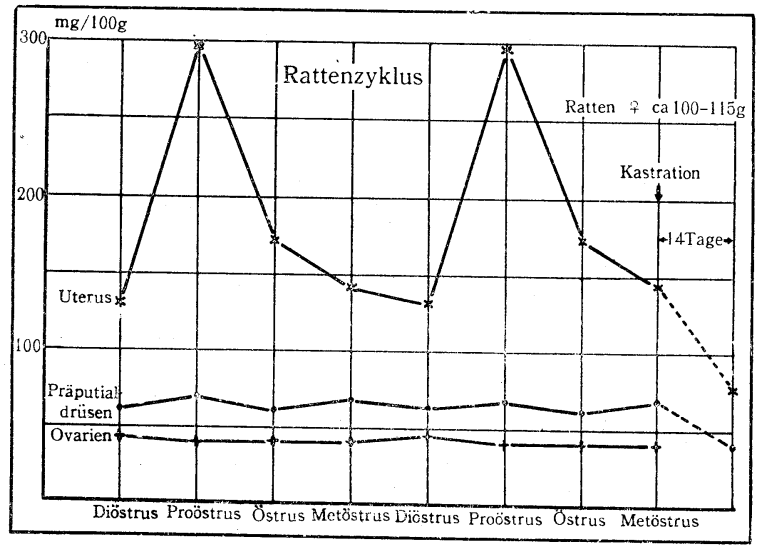

moeglich, einen mit dem Vaginalabstrich, oder mit den Schwankungen des Uterusgewichts parallel gehenden Gewichts anstieg und Gewichtsabfall nachzuweisen. Es liegt dies wohl daran, dass das Gewicht der Praeputialdruesen ein zu traeger Indikator fuer die Androgenproduktion der Ovarien ist. Sehen wir uns nun an, wie sich ein solcher androgener Zyklus in das rythmische Geschehen im Ovarium einordnen laesst. Darf ich Ihnen dazu nachfolgend eine Reihe von Schemata zeigen. Die naechste Abbildung 9 zeigt Ihnen den Erfolg einer Injektion von Choriongonadotropin an der infantilen

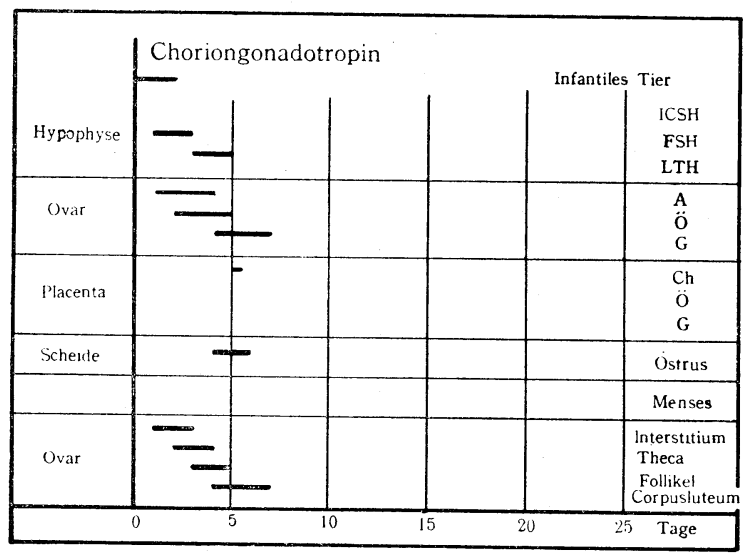

Ratte. Nach dieser Annahme wuerde der Injektion von Choriongonadotropin eine Sekretion von ICSH und diesem eine Ausschuettung von FSH in der Hypophyse nachfolgen und dieser wiederum eine Sekretion von lutsotropem Hormon, ausgeloest 
durch die im Ovarium unter diesem Einfluss aufgetretenen Sekretionen von Androgenen, Oestrogenen und Gestagenen.

Dementsprechend waeren auch die histologischen Veraenderungen im Ovarium in ihrer zeitlichen Aufeinanderfolge. Hypophysektomiert man, wie wir vor hnigehoert haben eine solche Ratte, so wuerde als einziges uebrig bleiben eine androgene Stimulierung der Ovarien mit der ihr entsprechenden Hypertrophie des Interstitiums, wil es aus der nächsten Abbildung hervorgeht (10). Danach wuerde sich uns der normale Zyclus in der Weise representieren, wie es die naechste Abbildung (11) zeigt. In der Hypophyse wuerde eine
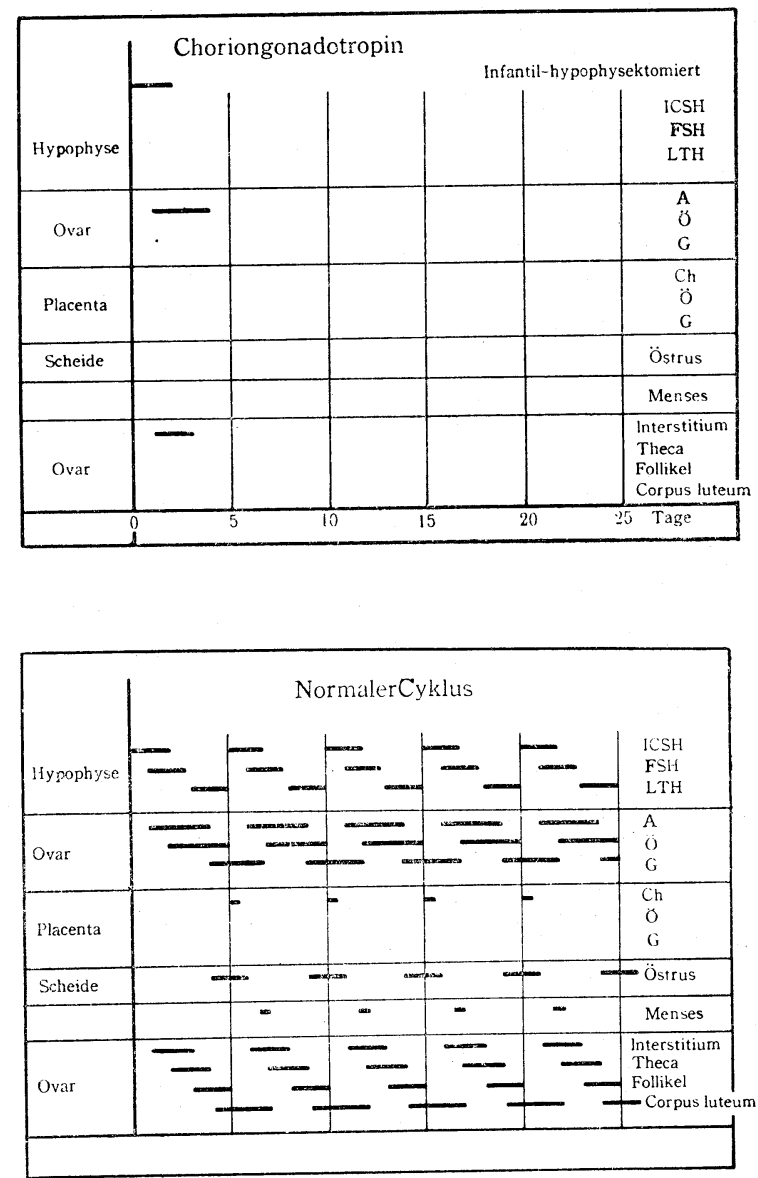

Sekretion von ICSH, FSH und luteotrophem Hormon aufeinandefolgen. Dementsprechen Ovar zunaechst eine primaere Sekretion von Androgenen, eine nachfolgende Sekretion von Oestrogenen, schliesslich eine solche von Gestagenen. Den einzelnen Sekretionen wuerde die zeitlich aufeinanderfolgende Hypertrophie des Interstitiums, der Follikel und des Luteingewebes entsprechen. Wir koennten nun den Zyclus stoeren, indem wir z.B. ein Follikelhormondepotpraeparat injizieren, wil es die nächste Abbildung besch- 
reibt (12). Dieses wuerde vom Zentrum aus nur die Sekretion von FSH hemmen und

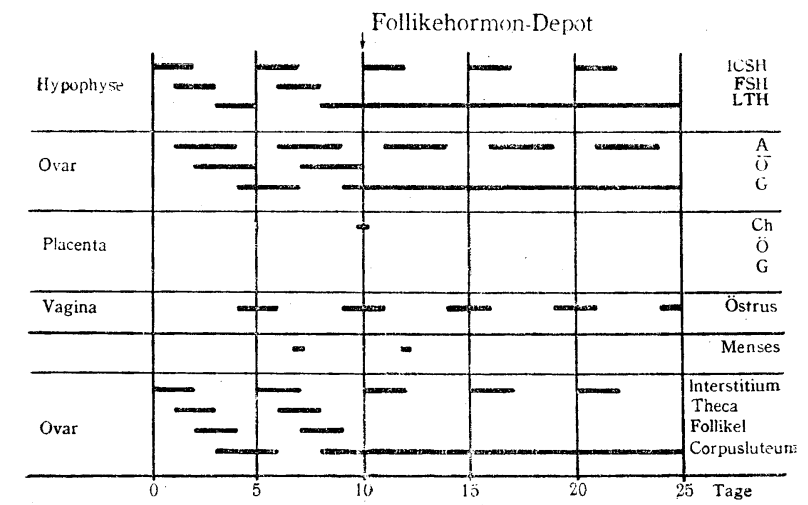

damit einen Teil der Hypophysensekretion lahmlegen, waehrend, wie wir vorhin gesehen haben, der Androgenzyclus weitergeht. Entsprechend dem bekannten Hohlwegeffekt wird dagegen unter Stimulierung der Luteotrophinsekretion der Hypophyse die Bildung von Gorpora lutea im Ovar angeregt. Dementsprchent wuerden wir dann im Ovarium eine leichte Interstitialzellenhypertrophie und andauernde Corporalutea finden. Schliesslich wuerde die Kastration durch Wegfall der hemmenden Hormone des Ovariums zu einer Ueberproduktion saemtlicher Hypophysenhormone fuehren, wie es uns die naechste Abbildung (13) vorfuehrt. Und als letztes wuerde sich die Schwangerschaft darstellen, wie es das naechste Bild zeigt. (14) Darf ich abschliessend noch die einzelnen Regulationen, wie sie sich nach diesen Gedankengaengen darstellen wuerden, in einem letzten Diapositiv (15) zeigen. Von einem Androgenzentrum ausgehend, wuerde in der Hypophyse die ICSH Produktion angeregt ICSH wuerde im Ovar einerseits zur Androgenbildung fuehren und andererseits ueber ein oestrogenes Zentrum

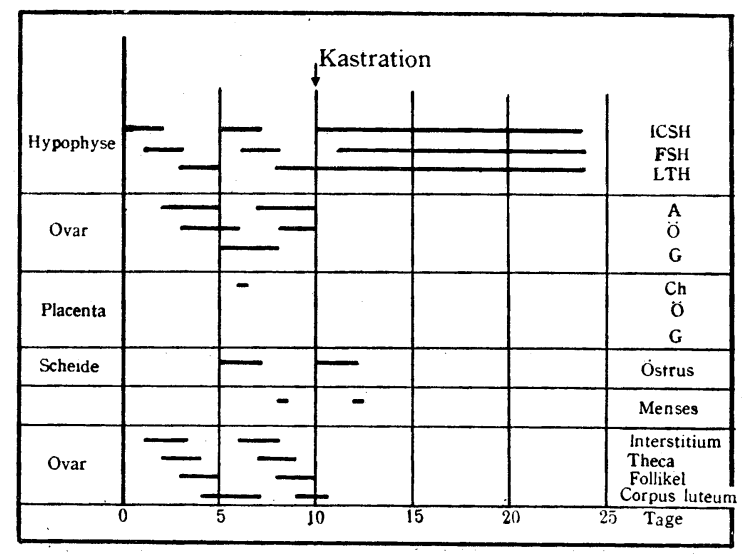

Vọl. 31 No. 5 

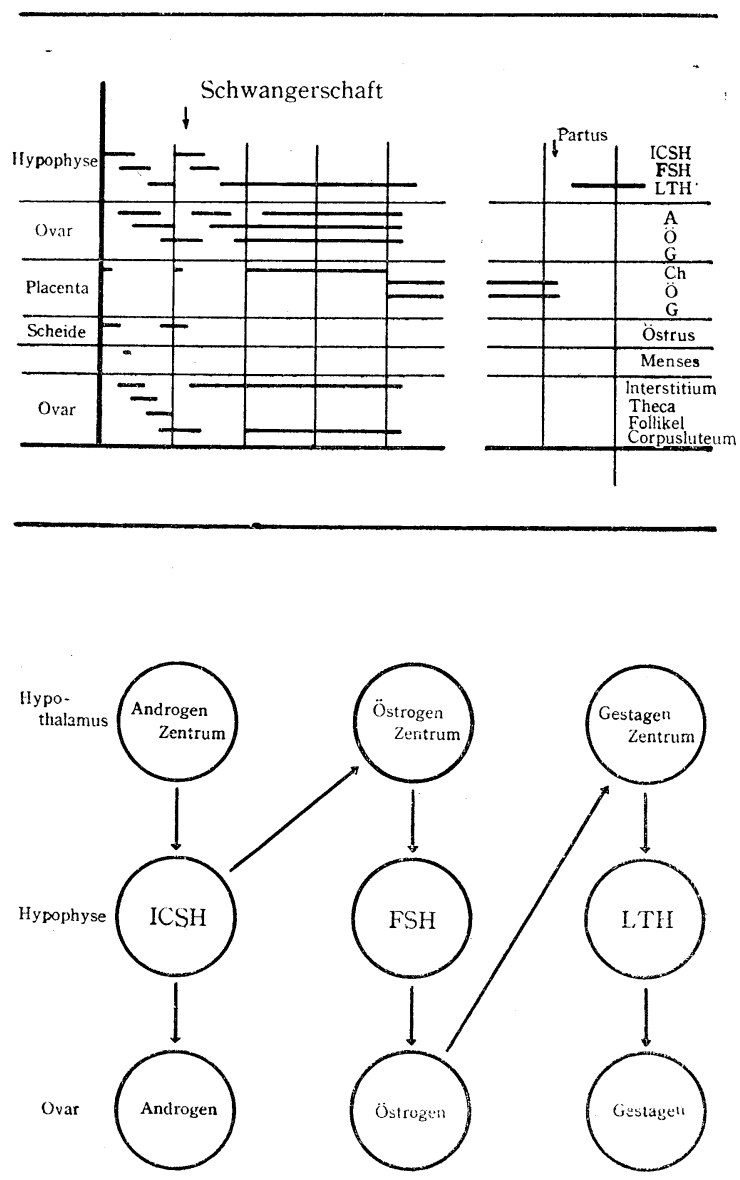

die FSH Sekretion anregen. Es its auch moeglich, dass ICSH die Hypophyse direkt zur FSH Sekretion anregt und diese beiden Hormone zusammen wuerden dann zur FollikeIreifung und zur Oestrogenbildung im Ovarium fuehren. Die Oestrogenbildung im Ovarium ihrerseits wuerde nunmehr ein gestagenes Zentrum oder die Hypophyse direkt anregen zur Luteotrophinbildung und damit zur Ausreifung des Corpus luteum fuehren. Die eingezeichneten Verbindungswege sind sicherlich nicht alle, und wir werden noch manche Regulationen kennen lernen. Die geschilderte Betrachtungsweise wuerde das Verhalten der hormonalen Vorgänge bei beiden Geschlechtern besser verstehen lassen und eine gleichsinnige Beeinflussung durch die hypophysaeren Gonadotropine bedeuten. ICSH, Chorion- und Stutenserumgonadotropin wirken am hypophysektomierten Maennchen die Androgenproduktion steigernd. Am normalen bewirken sie mit den Hypophysen-FSH auch spermiogenetisch und ancheinend die Androgenproduktion des Hodens erhoehend. Damit moechte ich meine Ausfuehrungen schliessen und Ihnen fuer Ihre Aufmerksamkeit danken. 